\title{
Examination of Industrial Design of Product Based on Combination of Interaction Analysis and TRIZ Tool -Take Example of Rescue Engineering Machinery
}

\author{
Sun Yuan ${ }^{1, a *}$, Jianjun Liu, ${ }^{1, b}$ and Mingliang Song ${ }^{1, c}$ \\ ${ }^{1}$ School of Architecture \& Fine Art, Dalian University of Technology, Dalian 116023, China \\ aidsun_yuan@126.com, bliujianjun@hotmail.de, ${ }^{\mathrm{C}}$ ammiyas@163.com
}

Keywords: Rescue Machine, Conceptual Design, Interaction Design, Ergonomics Design

\begin{abstract}
The environment after disasters is abominable and complicated. By increasing the wide adaptability of rescue machine to make it operate more quickly and flexibly and increase the efficiency of rescue operation. Based on investigating and analysis of the features of specific environment and functions of current existing engineering machines, we do analysis of industrial design on design elements like structure, function, dimension, color of the product. By combining Human-computer interaction analysis strategies and TRIZ conceptual design method, we present the concept of multifunctional rescue robot do the industrial design couple with simulation of the scheme by construction of computer-aided design system. We verify the effectiveness of this strategy by testing the completed product design.
\end{abstract}

\section{Introduction}

Due to the changing global climate and natural conditions, natural disasters are endangering life and properties of human being every moment. Current existing rescue machinery still cannot adapt to the complicated environment after the disaster. To significantly increase the efficiency of rescue operation, it is a proper method to combine specialized recuse machinery with machinery with universality. Product innovation is a series of processes consisting of problem analysis, concept formation and develop alternatives. The design behavior and thinking of industrial designer are extremely complex. As a strategy of defining the function of a product, interaction design can analyze the relationship between the product and the user and define the defination and priority of the functions at the stage of conceptual design of the product. TRIZ is the theory and method developed by the Soviet inventor ALTSHULLER for problem solving. It has been applied in lots of product development cases. By combining TRIZ tools and the strategy of interaction analysis, we can form a complete concept of industrial design of products.

\section{Research Method and Theory}

People-Centered Design - Interaction Analysis. People-centered design refers to design which is people oriented and serve the purpose of meeting the demands of the user. Interaction analysis is based on these three factors: people, machine and environment. And the relationships between the factors are people - to- people, people - to - machine, machine -to -machine, people - to environment, machine - to - environment, environment - to - environment [1]. Based on people-centered design, we develop new functions and use of the product by learning from related functions of other products. Besides, we can use new materials and technologies to increase product competitiveness by decreasing the cost and improve the quality refers to the theory of cost design. With the development of information and communication technology, the environment of knowledge society is changing. And the innovation mode of user-centered design based on user experience along with user participation is gradually formed.

The IFR Theory in TRIZ. IFR (Ideal Final Result) is a significant concept in TRIZ theory. IFR uses language which is unrelated to technology to describe the reason for innovation and it only focus on 
the requirement of user or function. Under the condition of IFR, the system will not take larger space. There is no extra mass, and no need of extra labor and extra maintainess. Only the useful functions will exist in the technical system while the useless and harmful functions will be eliminated[2]. Here are four feathers of IFR:

(1) It eliminates the deficiencies of the original system;

(2) It retains the merits of the original system;

(3) It will not make the system become more complicated;

(4) It will not create new deficiencies.

The important progress of innovation is acquired only based on deep understanding of the problems at current stage. It is important to make sure that the system works under the ideal components. During design process, the procedure of generation IFR from one aspect is called idealization. This process can be described by as the formula below:

Ideality $=\Sigma$ Benefits $/(\Sigma$ Costs $+\Sigma$ Harm $)$

The purposes of idealization are: Increase benefits; Decrease costs; Reduce Harm.

Visual Design. Visual design is the system identity design focusing on product design. It performs unified planning and designing on product design, design concept, technique, material, modeling, color, processing technology, packing, transportation, presentation, marketing techniques, advertising strategy and some other aspects. By doing that it can form a unified sensory and social identity of the product and enhance, shape and popularize the product identity. Product identity is the unified identity formed during the designing, developing, distributing and using. It is the result of the internal identity being integrated with the external visual identity of the product[3].

In the design of this product, by using the principles of system identity design, we focus on building the system of appearance design and surface decoration. The appearance system of the product mainly consists of the style of the appearance, material, color, surface processing, decoration and etc. Appearance design system is a major content of product identity design. By determining the suitable design language and style, we can develop series product and form our own unique style. Here are the requirements which the appearance design of a machinery product should meet:

a. The display and interface should be safe, practical. b. The shape, line, scale and color of the overall appearance of should be based on the aesthetic principles. It should be easy to maintain and service. It can help reduce the cost of production. It can fully express the design concept of the company. It should be consistent with the identity of the company while being unique.

The surface decoration system mainly consists of these components: company logo, standard font, standard color, nameplate, mark and etc. Surface decoration system is an indispensable and major content of product identity design. It presents the information of related companies by personalized combination and expression of those factors. Through that we can make the product identity be closely integrated with the enterprise image and constitutes an organic unity.

\section{The Concept Design Process Using Interaction Analysis and TRIZ Tool}

Design Goal. The deployment environment of the product is the disaster site somewhere after an earthquake. Earthquakes include tectonic earthquakes and induced earthquakes. Earthquakes will cause casualties and housing collapse, and it will indirectly lead to flood, fire, gas leak, plague and other disasters. And the road condition will be severe [4]. After analyzing the common features of the areas after earthquakes, here are the goals we set for the product:

The walking mechanism should have strong adaptability and can remove the barriers blocking traffic to make other specialized rescue vehicles able to enter the disaster site; It should have the function of life detection; It should be equipped with basic medical care equipments so that it would be able to do simple medical treatment to the ounded.

This product is designed for better adaptability to terrains after disaster in order to get to the disaster area as quickly as possible. Besides, by being integrated with functions of other machines which are commonly used for rescue operation after earthquakes, it will improve collaborative work and increase the efficiency of rescue operation. 
Interaction Analysis. The interaction analysis of rescue machinery is based on three factors: people, machine and environments. The relationships between the factors are people - to- people, people - to - machine, machine -to -machine, people - to - environment, machine - to - environment, environment - to - environment. Fig. 2 is the interaction phalanx diagram of rescue machinery. By studying the phalanx diagram, we can find out the relevance between human factor (user), mechanical factor (operation, power, energy, transmission, safety and main body), space factors and environmental factors. The larger the relevance between two factors, the closer they are related to each other on function and operation. And that is what we should pay more attention to while designing. During the stage of conceptual design, interaction analysis can help the designer clarify the relationships between the factors effectively and logically. Based on the association analysis of the design factors by using the interaction phalanx diagram, we can obtain clear goals and construct the man-machine relation of the product.

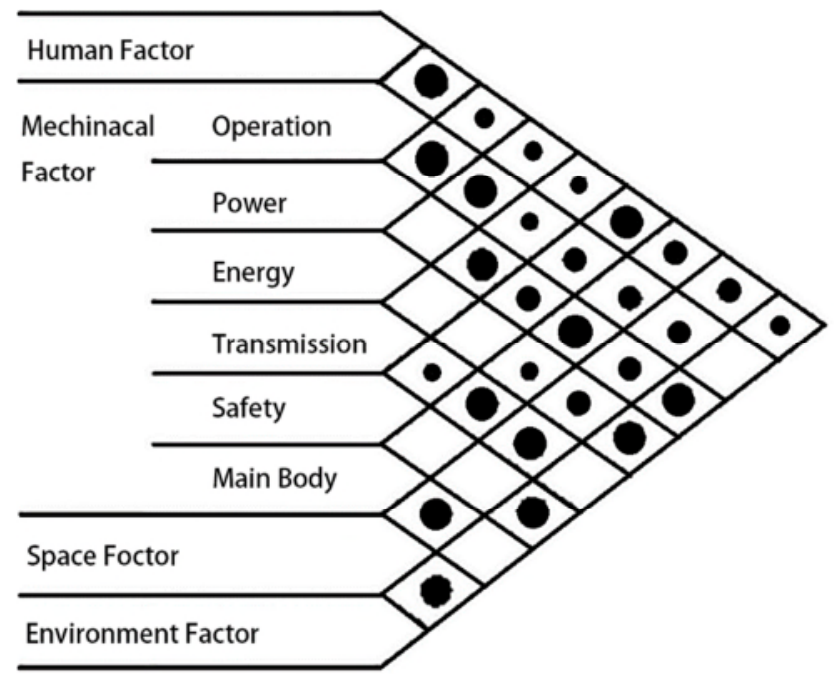

Fig. 1 Analysis phalanx diagram of product design factors.

Import TRIZ Tools. We propose a systematic and innovative method of design focus on the innovation of rescue product. In this paper, we take the advantages of TRIZ tools that can resolve the design conflicts systematically and innovatively and come up with the product design process framework shown in Fig. 1. Here are the procedures.First, demand analysis. We define design goals on specific demands and obtain the explicit input parameters of design. And we determine the optimal combination of the parameters by integrating analysis of factors including function, HMI, mentalness, finance and etc. Then we use contradiction matrix, effect knowledge base system or substance-field analysis to solve the problem based on the theory of IFR theory and knowledge base system. Evaluate the options generated and find the optimal solution.At last, on the basis of the optimal solution, we can do the conceptual design of the product and finish the sketch design and computer-aided design. Morphological Analysis. Modeling design of rescue product is based on its functions and it is a rational express of its functions. The modeling style should exhibit the characteristics of being safe, efficiently and reliable. The exterior design of rescue products includes these aspects: walking mechanism, turntable cover, manipulator and driver cab. Turntable cover and driver cab are the focus points during design. Turntable cover is a protective part. The internal composition consists of engine, tank, medical care equipment and etc. And the exterior design is even more closely related to product functions so we have to consider the influence of processes and operations on modeling. The exterior design should coordinate the relationships between people and machine and the relations between form, structure, function and operation of the product. It also should be in line with the physiological and psychological characteristics of the operator (human).

Color Analysis. As one of the function indicators of the product, commonly the main color of rescue products has great consistency. Usually it emphasizes safety and it is striking. Secondary colors and decorative lines are added depend on the exterior appearance of the product. We use proportional 
allocation and structure segmentation of the colors to perfect the visual experience that cannot be expressed by the modeling of the product. And we can achieve the functional expression of the product in a foreseeable and controllable manner. Color is the first visual experience of the product and it indicates the function of the product.

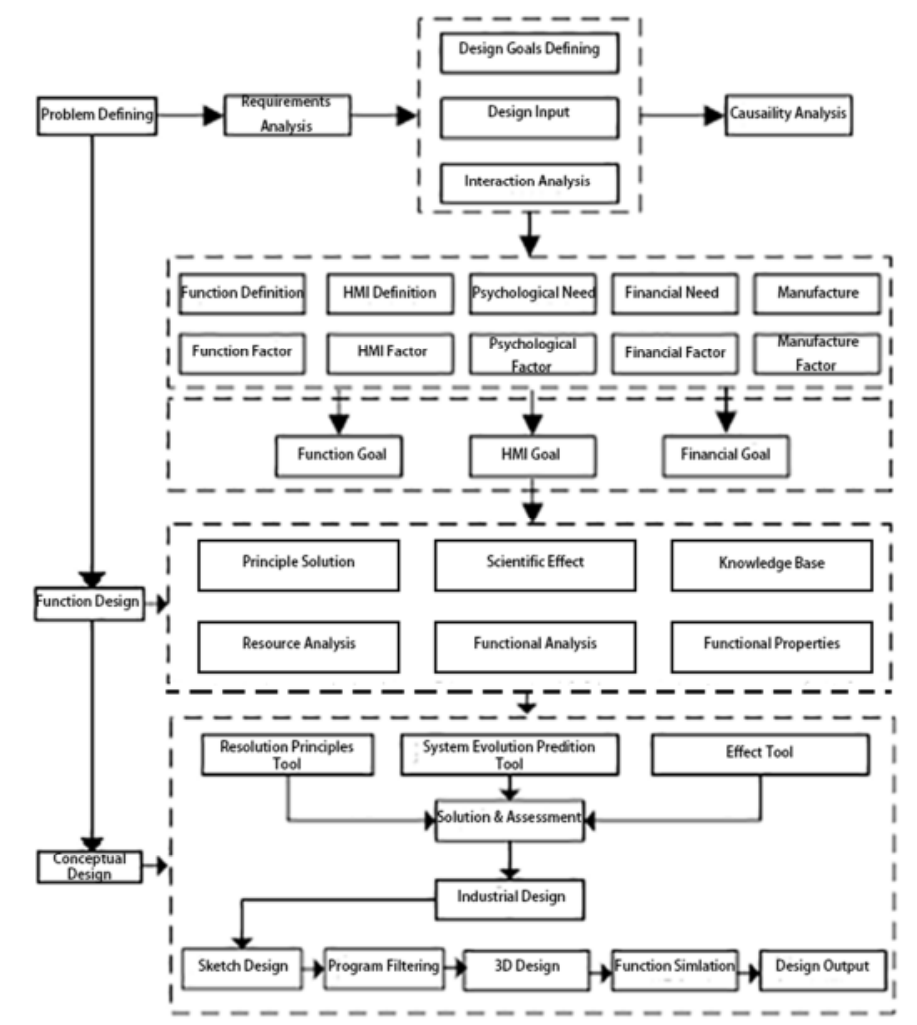

Fig. 2 Conceptual design process framework model using triz tool.

\section{Design Scheme Expansion}

Conceptual Design. Sketch is a method used to easily present the structure design and the features of appearance design [5]. The initial sketch of this rescue machine is shown in Fig. 3. We use strong shape symbols to highlight that this machine plump and ably. The closed shape outstands the integrity of the turntable. Considering safety problem, we simplify the complex structures inside the turntable and place them inside the machine. For the hood, we use four splines connected end to end and closed as boundaries and two internal fitting splines to form a smooth and elegant shape. We choose triangular pedtrail wheel system as the walking mechanism of this machine. It is equipped with two robotic arms and a dozer blade used to eliminate barriers below the arms. Inside the turntable there are engine, hydraulic system and medical aid kit. Driver cab can switch positions easily at working condition for the multiple DOF design. After confirming the design of mechanism andappearance of the product, we build a digital model of the product by using Rhino+Keyshot, a computer-aided industrial design software.

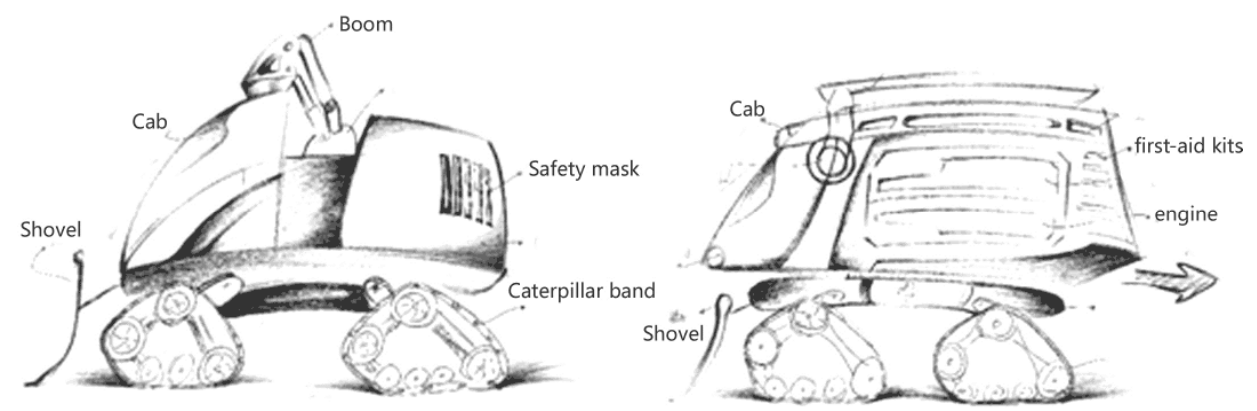

Fig. 3 Initial sketch of the rescue machine. 

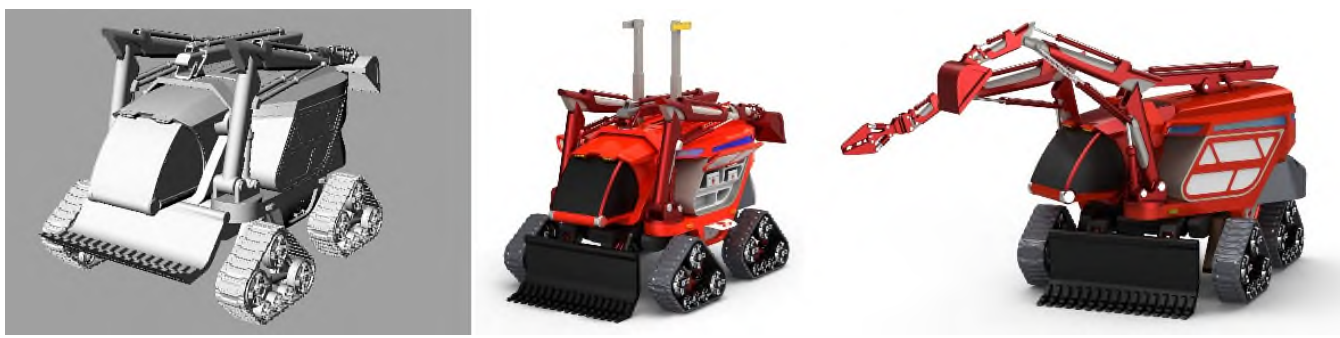

Fig. 4 Simulation of the final design.

Product Realistic Rendering. After finishing the digital model of product by using Rhino, we set the virtual material set in Keyshot and render the model. Based on principles of photography, we set the light source, material and position of the camera and coordinate with the model to make the model look more realistic in lighting and material in the virtual environment [6].

Product Material Expression:Material expression is the key to make a virtual product look more realistic. To reproduce the effect of realistic material, we have to use the physical parameters of real materials and keep adjusting the settings according to the render view of the virtual camera.

In this product, the materials we used consist of plastic, metallic paint, highly reflective metal and so on. Depending on the light source characteristics of different materials, we adjust the parameters like diffusion, refection, glossiness and subdivision to acquire a realistic effect shown in Table 1 below.

Table 1. Setting parameter of basic material attribute.

\begin{tabular}{c|c|c|c}
\hline Parameter & Metal Paint & Plastic & Metal \\
\hline Diffuse(RGB) & 245/16/16 & $163 / 163 / 163$ & $62 / 62 / 62$ \\
\hline Reflect & Falloff & 1.0 & 1.0 \\
\hline Refl.glossiness & 0.9 & 0.8 & 0.85 \\
\hline Subdivs & 16 & 8 & 8 \\
\hline
\end{tabular}

Virtual Display Environment. Creating a realistic display environment can present the characteristics of the virtual product and make it look more realistic. The reproduction of the scene we should consider the functions and environment of use of the product. Typical environments include disaster area after earthquake, traffic blocking after disaster and so on. The scene file is BMP and its resolution should meet the accuracy requirements of the output.

Virtual Light Parameter Selection. Adding lighting effect in the CAD virtual environment can enhance the photorealistic of the product. It can highlight the product and present the environment better. Optional virtual lights include ambient light, spotlight and floodlight. We can achieve the desired effects by adjusting the relative positions of virtual light source to the product and the parameters of light properties. The settings of color, brightness and range will directly influence the degree of detail, color variation and modeling of the product.

Product Visual Design. The basic elements of visual design of the body consist of graphics, text and logo. We import the basic graphics of graphic design into the CAD virtual environment and coordinate them to calibrate with the product scale. 2D drawing should strictly follow the scale of the body. After coloring we import the file and adjust the coordinates of the $2 \mathrm{D}$ graphics on 3D surface. The last step is color rendering.

On color design of the product, there are several principles we follow while considering the characteristics of the product:

(1) Visual Balance Principle: Balance refers to the condition that more than two colors are visually smooth, steady and equal when they are collocated. Level of lightness, chroma and size ratio are the factors affect the color balance. In this product we determine to use red and grey to achieve a equalized layout.

(2) Emphasis Principle: When collocating more than two colors, we have to considering the overall effect of the product. We should adjust the lightness, chroma and area of colors according to the relationship between main color, secondary color and collocative color. During coloring scheme design, we first choose the outline color based on the structure layout features of aerial vehicle and 
add some appropriative changes to avoid being monotonous on the basis of main body being stressed out.

(3) Color Harmony Principle: Adding a color with commonality in the colors used in the product is a visual transition and connection of parts of different colors look unified and harmonious. And we use this color as the main color of the product for its visual controlling effect. In this product we use red as main color.

Final Design Expression. The final design is shown in Fig. 4. The main purpose of this rescue machine is to execute maximum rescue operation as a single rescue vehicle. By integrating the functions of currently existing machinery, the product can cooperate more coordinatively and increase the efficiency of rescue operation. We choose mining function, cutting function and dozer function as the main functions of the rescue engineering machine. And we choose triangular pedtrail mechanism as walking mechanism to deal with the complex terrain conditions after earthquakes. There are two inflatable tents on both sides of this machine which can be used as temporary relief camps. And the LED tower on top of the vehicle can provide lighting for people around.

\section{Conclusion}

With the continuous deterioration of the global climate and environment, natural disasters and man-made disasters continue to harm lives of ordinary people. Buildings collapse causes not only huge economic losses and casualty but also irreparable psychological pain Immediate rescue is the most important need when disasters occur. Only by using specialized equipment, scientific distribution and completed placement system we can heal the wounds of the survivors. This rescue engineering machine is designed in the basis of fully analysis of characteristics of domestic geological disasters along integrated with the mature technologies of foreign civil and military rescue machinery. And we take full consideration of the objective reality on pattern, function, color and etc.

\section{References}

[1] M. Takahashi, From Idea to Product-The Integrated Design Process, Hong Kong: Hong Kong Productivity Council, 1994.

[2] Y. S. Liao, The Research of Innovation in Industrial Design based on the IFR in TRIZ, Art Des. (5) (2007) 125-126.

[3] H. F. Ding, Z. M. Feng, H. Y. Fu, Conceptual Design of an Exactly Straight Lifting Forging Manipulator, ASME J. Mech. Robotic. 6(3) (2014) 034502.

[4] Y. Wang, C. G. Gu, Thoughts about Emergency Rescue after Earthquake Disasters, Fire Tech. Prod. Inform. (8) (2011) 20-22.

[5] S. D. Savransky, Engineering of creativity, NewYork: CRC Press, 2000.

[6] P. Y. Wu, H. L. Wang, Analysis of Domestic and Foreign Emergency Rescue Capabilities of Major Disasters, Fire Tech. Prod. Inform. (2) (2012) 23-26.

[7] J. S. Gero, Artificial intelligence and creativity, Norwell: Kluwer Academic Publishers, 1994. 